\title{
Persistence of Long-Term Memory in Vitrified and Revived Caenorhabditis elegans
}

\author{
Natasha Vita-More ${ }^{1,2}$ and Daniel Barranco $0^{1,3}$
}

\begin{abstract}
Can memory be retained after cryopreservation? Our research has attempted to answer this long-standing question by using the nematode worm Caenorhabditis elegans, a well-known model organism for biological research that has generated revolutionary findings but has not been tested for memory retention after cryopreservation. Our study's goal was to test $C$. elegans' memory recall after vitrification and reviving. Using a method of sensory imprinting in the young $C$. elegans, we establish that learning acquired through olfactory cues shapes the animal's behavior and the learning is retained at the adult stage after vitrification. Our research method included olfactory imprinting with the chemical benzaldehyde $\left(\mathrm{C}_{6} \mathrm{H}_{5} \mathrm{CHO}\right)$ for phase-sense olfactory imprinting at the L1 stage, the fast-cooling SafeSpeed method for vitrification at the L2 stage, reviving, and a chemotaxis assay for testing memory retention of learning at the adult stage. Our results in testing memory retention after cryopreservation show that the mechanisms that regulate the odorant imprinting (a form of longterm memory) in $C$. elegans have not been modified by the process of vitrification or by slow freezing.
\end{abstract}

\section{Introduction}

C aenorhabditis elegans IS ONE OF THE MOST IMPORTANT models used in biology and neurology ${ }^{1}$ and has countless applications in the area of biological sciences. ${ }^{2-4}$ The simplicity of its size $(1 \mathrm{~mm})$, the transparency of its neuronal network (hermaphrodites contain 302 neurons) ${ }^{5}$ and its short but complex life cycle make $C$. elegans of potential value to studies of memory retention after cryopreservation. Through the use of $C$. elegans as a model, numerous experiments have been performed in the area of memory and neurodegenerative diseases, ${ }^{3,4}$ many of which explain the mechanism of memory, including the differences between short- and long-term memory. ${ }^{6-13}$

Our study addresses the specific interest that long- or short-term memories of cryopreserved and revived animals have not been tested. The organism of choice to explore this question is $C$. elegans because it is the only organism in which both cryopreservation and revival have been demonstrated and there is a well-defined assay of learning.

To evaluate our study's specific interest, we combined and used three principal methodologies developed previously: (1) Olfactory imprinting for C. elegans, ${ }^{9}$ (2) vitrification of $C$. elegans by the SafeSpeed cryopreservation protocol, ${ }^{14}$ and (3) a chemotaxis migration assay. ${ }^{9}$ Attached to this project and as an extra goal, we also evaluated the persistence of memory using the traditional protocol of cryopreservation of $C$. elegans by slow freezing. ${ }^{2}$

Our research has established a protocol for examining the results of olfactory imprinting, demonstrating long-term memory retention of this simple adult animal after vitrification and reviving. This study provides new knowledge to the field of cryopreservation, and we consider such findings to be a preliminary step in ascertaining the preservation of memory of simple animals undergoing vitrification.

\section{Learning in C. elegans}

In our study, we first explored learning through chemical attractants for testing memory of behavior performance. In diverse environments, $C$. elegans have been shown to habituate to learned smells and to associate attractant and aversive chemicals with the presence or absence of food. ${ }^{9,11,12}$ One method for training and testing learning in the worms is to expose them to a specific chemical compound over a restricted period of time with the presence or absence of food to create a pattern of behavior. When encountering the chemical compound at later times, the worms' level of

${ }^{1}$ Alcor Research Center (ARC), Alcor Life Extension Foundation, Scottsdale, Arizona.

${ }^{2}$ University of Advancing Technology, Tempe, Arizona.

${ }^{3}$ CryoBioTech. Engineering School, University of Seville, Seville, Spain.

(C) Vita-More and Barranco 2015; Published by Mary Ann Liebert, Inc. This Open Access article is distributed under the terms of the Creative Commons Attribution Noncommercial License (http://creativecommons.org/licenses/by-nc/4.0/) which permits any noncommercial use, distribution, and reproduction in any medium, provided the original author(s) and the source are credited. 
response (memory retention) is tested and evaluated by a chemotaxis migration assay. ${ }^{9,12}$ In their findings of associative short-term memory, Kauffman et al. ${ }^{12}$ showed that after $C$. elegans are exposed to the chemoattractant butanone, the worms' memory response starts to decrease $1 \mathrm{hr}$ after exposure. In their findings on long-term memory, Remy and Hobert ${ }^{9}$ showed that after C. elegans are exposed to attractant chemicals such as benzaldehyde at the early larval stage, the worms' memory response in the adult stage continues to be retained after 5 days, or $120 \mathrm{hr}$, for phasesense learning, referred to as imprinting.

Imprinting is one of the mechanisms key to analyzing basic neural aspects of memory ${ }^{15}$ and is known as a type of learning in which young organisms make strong associations. Because of the potential for strong associations, imprinting is often used to explain early adaptation or bonding to particular sensory cues, such as olfactory signals. Olfactory-based imprinting has been studied in many species, including humans, other primates, mammals, ungulates, aquatic animals, birds, and microscopic simple animals (such as C. elegans). In olfactory imprinting, ${ }^{9}$ there is a distinct period of time (a window of opportunity or physiological state that lasts for a limited time) in which an organism can develop a long-lasting learned response. Notably, the distinct period of time distinguishes this form of memory from other types of learned olfactory adaptive procedures in C. elegans that only last for a limited time and in which olfactory imprinting behavior is mediated by a single pair of interneurons. ${ }^{9}$ Moreover, there is evidence that olfactory imprinting of $C$. elegans is transmitted to the next generation if the imprint is accomplished successfully for more than four generations. ${ }^{16}$

\section{Cryopreservation of C. elegans}

There are two traditional ways to cryopreserve biological samples: (1) Slow freezing and (2) vitrification. ${ }^{17,18}$ These cryopreservation methods are differentiated by the cooling and warming rates and the concentration of cryoprotectant used to obtain successful cryopreservation, whether or not ice crystals are formed, and the attendant dehydration of the cells.

Slow freezing requires a low concentration of cryoprotectant and slow cooling and warming rates with the formation of ice crystals. Although this method has been the traditional protocol for cryopreserving C. elegans, it has a limited survival rate of around $25 \%-35 \%$ for the L1 and L2 stages. ${ }^{2}$

Vitrification is the transformation of a liquid substance into a vitreous solution, achieved by high cooling rates and concentrations of cryoprotectant, eliminating ice formation. ${ }^{18-20}$ The basic method for vitrifying cells is known as Cryotop, which has been used for embryos and oocytes for over a decade with excellent results on the basis of survival rates and is considered to be the most reliable cryopreservation method for cells and embryos. ${ }^{21,22}$ The Cryotop vitrification protocol directly submerges cells into liquid nitrogen. This method developed from minimum volume cooling $(\mathrm{MVC})^{23}$ using traditional sample straws ${ }^{24}$ and proved to have a higher survival rate for cells as compared to conventional vitrification, due to the much higher cooling rates attainable with small volumes.

Combining the thermal performance of quartz capillaries, ultra-fast cooling-rates (slush nitrogen), ${ }^{19,25,26}$ and MVC, ${ }^{22}$ the protocol for vitrification of C. elegans in the L1 and L2 stages was developed. ${ }^{26}$ However, the warming rate in this protocol was too slow to successfully achieve vitrification of other stages of the larvae and the adult. Recent studies concerning the importance of the warming rates, more than the cooling rates, ${ }^{27-30}$ have been contributing factors in developing new techniques to improve vitrification protocols.

The SafeSpeed closed device ${ }^{31}$ is a new technology based on ultra-fast warming rates that was originally developed to revive vitrified oocytes and embryos and offers an alternative for vitrifying $C$. elegans, simplifying the protocol and ensuring survival rates $\sim 100 \%$ for all $C$. elegans stages, including the adult stage. ${ }^{14}$ This method also presents an excellent alternative for cryopreserving $C$. elegans, with much better survival rates (for all stages) than slow freezing and is an easier protocol than vitrification using quartz capillaries. $^{14,25}$

\section{Materials and Methods}

\section{C. elegans native strain}

Worm maintenance, including breeding, was conducted as described by Stiernagle. ${ }^{32}$ The Wild isolate Bristol strain N2 was obtained from the Caenorhabditis Genetics Center (CGC) at the University of Minnesota.

\section{Control group}

The control group consisted of eight sets of 100 or more worms for each study. We used a combination of variables in each study to observe and identify the native behavior of the worms in the chemotaxis assay and the effects of cryoprotectant solutions and cryopreservation protocols on the worms' behavior. The eight sets of studies are identified as S1, S2, S3, S4, S5, S7, S8, and S9 (Table 1).

We applied the term "untrained" to sets of worms that were not imprinted with benzaldehyde and the term "trained" to sets of worms that were imprinted with benzaldehyde. Studies included assembling of trained and untrained worms and the presence or absence of a cryopreservation protocol. First, trained and untrained worms were exposed to either the cryoprotectant vitrification solution or the cryoprotectant slow freezing solution; and, second, the cryopreservation protocols were completed by vitrification or by slow freezing (Table 1).

\section{Experimental group}

We describe the experimental group as two sets of 100 or more worms for each study. The worms were trained, cryopreserved, revived, and tested for memory recall. The cryopreservation methods included worms that were trained and cryopreserved by vitrification and worms that were trained and cryopreserved by slow freezing. This was the most important study to test the retention of memory after vitrification or slow freezing. The two sets of studies are identified as S6 and S10 (Table 1).

\section{Phase-sense olfactory imprinting}

Using the Remy and Hobert ${ }^{9}$ method for olfactory imprinting, $20 \mathrm{~L} 1$ stage worms were pipetted into a $6-\mathrm{cm}$ petri dish with a lawn of Escherichia coli OP50 and exposed to 
Table 1. Description of the Studies

\begin{tabular}{|c|c|c|c|c|c|c|}
\hline & \multicolumn{2}{|c|}{ Olfactory imprinting } & \multicolumn{2}{|c|}{ Cryoprotectant solutions } & \multicolumn{2}{|c|}{ Cryopreservation } \\
\hline & $\begin{array}{c}\text { Trained } \\
\text { (benzaldehyde) }\end{array}$ & $\begin{array}{c}\text { Untrained } \\
\text { (distilled water) }\end{array}$ & $\begin{array}{l}\text { Vitrification } \\
\text { solution }\end{array}$ & $\begin{array}{c}\text { Slow freezing } \\
\text { solution }\end{array}$ & Vitrification & $\begin{array}{c}\text { Slow } \\
\text { freezing }\end{array}$ \\
\hline \multicolumn{7}{|c|}{ Control group } \\
\hline Study 1 & No & Yes & No & No & No & No \\
\hline Study 2 & Yes & No & No & No & No & No \\
\hline Study 3 & No & Yes & Yes & No & No & No \\
\hline Study 4 & Yes & No & Yes & No & No & No \\
\hline Study 5 & No & Yes & Yes & No & Yes & No \\
\hline Study 7 & No & Yes & No & Yes & No & No \\
\hline Study 8 & Yes & No & No & Yes & No & No \\
\hline Study 9 & No & Yes & No & Yes & No & Yes \\
\hline \multicolumn{7}{|c|}{ Experimental group } \\
\hline Study 6 & Yes & No & Yes & No & Yes & No \\
\hline Study 10 & Yes & No & No & Yes & No & Yes \\
\hline
\end{tabular}

The composition of each study is the result of the combination of three different methods-olfactory imprinting (trained or untrained worms), the use of cryoprotectant solutions (vitrification solution or slow freezing solution), and cryopreservation (vitrification or slow freezing).

the attractant benzaldehyde, applied by swiping $4 \mu \mathrm{L}$ of $1 \%$ benzaldehyde on the inside of the petri dish lid every hour for $8 \mathrm{hr}$. At the end of the $8-\mathrm{hr}$ treatment period, the petri dish lid was replaced with a clean, unswiped lid. For untrained worms, the protocol was the same but we swiped $4 \mu \mathrm{L}$ of distilled water over the petri dish lid.

\section{Slow freezing for $\mathrm{C}$. elegans}

Cryopreservation by slow freezing was performed 1 day after olfactory imprinting. Using the method of Brenner, ${ }^{2}$ the worms at the L2 and L3 stages were introduced into a cryovial (cryogenic tube) with the traditional mix of cryoprotectant for slow freezing at $15 \%$ vol/vol glycerol in M9 Buffer ( 3 grams $\mathrm{KH}_{2} \mathrm{PO}_{4}, 6$ grams $\mathrm{Na}_{2} \mathrm{HPO}_{4}, 5$ grams $\mathrm{NaCl}$, $1 \mathrm{~mL}$ of $1 \mathrm{M} \mathrm{MgSO}_{4}, \mathrm{H}_{2} \mathrm{O}$ to 1 liter). The cryovials were transferred to a $-80^{\circ} \mathrm{C}$ freezer for 2 weeks. After 2 weeks, the worms were transferred to a petri dish with a lawn of Escherichia coli OP50.

\section{SafeSpeed for C. elegans}

One day after olfactory imprinting, the worms were vitrified. We used the SafeSpeed C. elegans protocol described in Gallardo et al. ${ }^{14,33}$ This method consists of two components: (1) SafeSpeed closed device (container for the worms), and (2) SafeSpeed closed system vitrification and warming media (cryoprotectant solutions). For each vitrification study using the SafeSpeed closed device, we vitrified five sets of worms at the L2 and L3 stages. The protocol started by transferring the worms from the petri dish to $50 \mu \mathrm{L}$ of Washing Solution (WS) for 2 min using a Pasteur pipette. The worms were then transferred to $100 \mu \mathrm{L}$ of Equilibration Solution (ES) for $10 \mathrm{~min}$. Later, the worms were placed in $100 \mu \mathrm{L}$ of Vitrification Solution (VS) for $1 \mathrm{~min}$, and then introduced into the SafeSpeed closed device. The device was closed with a heat-sealer to avoid contamination and then quickly immersed in liquid nitrogen. The device was held in liquid nitrogen for $30 \mathrm{~min}$ and rewarmed in a $37^{\circ} \mathrm{C}$ water bath. The SafeSpeed closed device was opened with scissors, and the worms were placed into
$200 \mu \mathrm{L}$ of Thawing Solution (TS) at $37^{\circ} \mathrm{C}$ for $1 \mathrm{~min}$ and then quickly transferred to $200 \mu \mathrm{L}$ of the Diluent Solution (DS) for $3 \mathrm{~min}$. To finish this protocol, the worms were washed twice in two drops of $100 \mu \mathrm{L}$ of WS, with $5 \mathrm{~min}$ for the first wash and $3 \mathrm{~min}$ for the second wash, and finally the worms were transferred to a petri dish with a lawn of E. coli OP50.

\section{Chemotaxis assays}

The odorant chemotaxis assays were performed on worms at the adult stage, 5 days after olfactory imprinting. ${ }^{9} \mathrm{We}$ used $12 \times 12-\mathrm{cm}$ square low-salt agar plates $(2 \%$ agar, $5 \mathrm{mM}$ $\mathrm{KPO}_{4}, \mathrm{pH} 6,1 \mathrm{mM} \mathrm{MgCl}$, and $1 \mathrm{mM} \mathrm{CaCl}_{2}$ ). On the outside bottom of each square dish, we drew eleven lines, marking off an area of $1 \times 12 \mathrm{~cm}^{2}$ and assigned values from -6 to +6 . In the first delineated area of the plate (with value $-6)$, three drops ( $\mu \mathrm{L}$ each) of $1 \mathrm{M}$ sodium azide were placed in the agar with equal spacing. In the same area and in the same position as the drops of sodium azide, but in the lid of the square dish, three drops of $\mathrm{H}_{2} \mathrm{O}_{\mathrm{d}}(4 \mu \mathrm{L}$ each) were placed. On the other side of the square dish, in the area with value 6 , the same drops $(1 \mu \mathrm{L}$ each) of $1 \mathrm{M}$ sodium azide were placed; however, the only difference was that on the lid of the dish we put three drops of $1 \%$ benzaldehyde (1/100 diluted in $\mathrm{H}_{2} \mathrm{O}_{\mathrm{d}}$ ) (see Supplementary Figure 1 in Remy and Hobert ${ }^{9}$ ).

We used a platinum wire to select and pick up individual worms from a petri dish with $E$. coli OP50, transferred the worms to a petri dish without food, and held them for $15 \mathrm{~min}$. Then, 20 worms were transferred along the centerline of the square dish. The worms were counted every $15 \mathrm{~min}$. At $1 \mathrm{hr}$, we had accumulated 80 values (each value designated the area where was the worms were in that moment), and we calculated a migration report, which Remy and Hobert ${ }^{9}$ referred to as the migration index (MI).

\section{Statistical analysis}

We used the software SPSS, version 20, to analyze the data set. The mean and the standard error of the variable MI 
TABle 2. Number of Worms For Each Type of Study and Migration Index Mean

\begin{tabular}{rrrrrr}
\hline$S$ & $N W$ & $N C A$ & $M I$ & $S E$ & $\mathrm{p}$ \\
\hline 1 & 110 & 6 & 1.34 & 0.36 & 0.00 \\
2 & 169 & 11 & 4.23 & 0.21 & \\
3 & 115 & 7 & 2.00 & 0.38 & \\
4 & 121 & 6 & 3.75 & 0.13 & \\
5 & 122 & 8 & 1.62 & 0.15 & \\
6 & 128 & 8 & 3.51 & 0.11 & \\
7 & 108 & 6 & 1.51 & 0.23 & \\
8 & 115 & 6 & 3.91 & 0.18 & \\
9 & 114 & 6 & 1.73 & 0.25 & \\
10 & 118 & 6 & 3.37 & 0.13 & \\
\hline
\end{tabular}

Type of study (S), number of worms (NW), number of chemotaxis assay (NCA), migration index mean (MI), standard error (SE), and $p$ value on the analysis of co-variance (ANOVA) test $(p)$.

Types of studies (S) from 1 through 10: S1, untrained and not vitrified; S2, trained and not vitrified; S3, untrained, not vitrified and cryoprotectant solution; $\mathrm{S} 4$, trained, not vitrified and cryoprotectant solution; S5, untrained and vitrified; S6, trained and vitrified; S7, untrained, no slow freezing and cryoprotectant solution; S8, trained, no slow freezing and cryoprotectant solution; S9, untrained and slow freezing; and S10, trained and slow freezing.

were calculated for each type of study (Table 2), and to compare each study, different tests were applied over this variable (MI). The homogeneity of the variance was tested with the Levene test and the mean was compared with the one-factor analysis of variance (ANOVA) test. Finally, the Tahame test was used to test equality between pairs of study analyses.

\section{Results}

Cryopreservation of C. elegans: Slow freezing and SafeSpeed for C. elegans

The survival rates for our study did not show deviation from the expected original slow freezing method of Brenner $^{2}$ or the SafeSpeed method of Gallardo et al. ${ }^{14,33}$ The survival rate for slow freezing with L2-L3 worms was $\sim 20 \%$, and for vitrification was $\sim 100 \%$.

\section{Chemotaxis assays and migration index}

The trained worms (worms imprinted with benzaldehyde) preferred areas 5 and 6 on the square petri dish, close to the benzaldehyde drops, and the untrained worms preferred areas 1 and 2, demonstrating a native attraction to the benzaldehyde (Fig. 1). The mean of the MI was higher and very similar in all the studies with trained worms. The studies with untrained worms were also similar (Fig. 1). The highest value of the MI was 4.23 and was obtained with trained and unvitrified worms and the lowest value was 1.34 in the untrained and not vitrified worms (Table 2). In general, the response of the trained worms to the benzaldehyde was double that of the untrained worms, whether they were cryopreserved or not. The variance of MI did not show homogeneity $($ Levene $=2.920, \mathrm{df} 1=9$, df $2=60, p=0.006$ ) and the comparison of the media through one-factor ANOVA showed differences between groups $(F=26.061$, $p=0.000$ ). The Tahame test, for comparisons in pairs, showed differences between the two groups-trained and untrained worms - and no differences between the studies inside of each group.

There were no differences between trained and vitrified worms and trained and not vitrified worms (Tahame, $i-j=0.72$,

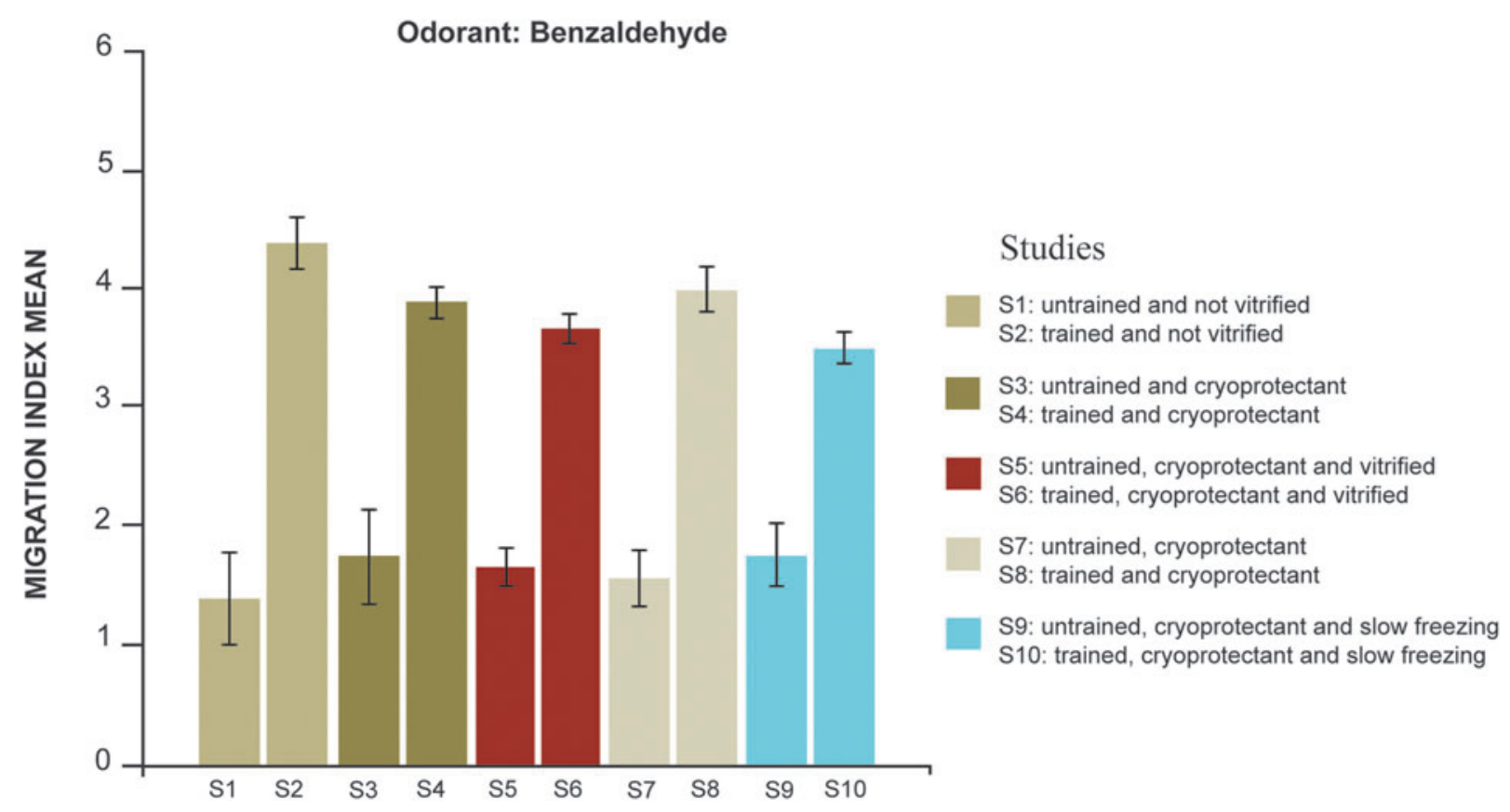

FIG. 1. Explanation of migration of untrained and trained worm studies: S1 (untrained and not vitrified) and S2 (trained and not vitrified); S3 (untrained and cryoprotectant) and S4 (trained and cryoprotectant); S5 (untrained, cryoprotectant and vitrified) and S6 (trained, cryoprotectant and vitrified); S7 (untrained and cryoprotectant) and S8 (trained and cryoprotectant); S9 (untrained, cryoprotectant and slow freezing) and S10 (trained, cryoprotectant and slow freezing). 
$p=0.305)$. Also, there were no differences between untrained and slow freezing and trained and slow freezing (Tahame, $i-j=0.24, p=0.138$ ). Both methods of cryopreservation did not show differences in the MI average of trained worms and were also similar to the MI of trained and worms that were not cryopreserved (Fig.1).

\section{Discussion}

The study shows the first results related to the persistence of memory after vitrification. Prior to this study, there had been no other study or methodology to carry out this project or a similar project. The protocols that permitted us to examine memory in $C$. elegans were developed during the last 10 years, $, 11,12$ and the protocols for successful vitrification of $C$. elegans with high survival rates were developed over the past few years. ${ }^{26,32}$ C. elegans is one of the first animals that has been cryopreserved and revived by a completely artificial process $2,26,32$ and is also one of the most important model organisms to be used in biology.

We used the $C$. elegans olfactory imprinting method of Remy and Hobert ${ }^{9}$ in this study because it allows sufficient time to carry out all the steps in our cryopreservation protocol. The period between the olfactory imprinting (at the L1 stage) and the evaluation of memory in the MI assay (at the adult stage) is more than $26 \mathrm{hr}$. Specifically, the cryopreservation protocol in our study continues over 5 days. During this time, it is necessary to allow 30-45 min for vitrification processes, 15-30 min for slow freezing processes, and $24 \mathrm{hr}$ for all processes to safely recover and verify the survival of the worms. Other methods ${ }^{12}$ for testing memory in C. elegans where the time frame is not enough to allow for cryopreservation and recovery of the worms were discarded. Additions to our study might include testing the $t t x$ - 3 and $s r a$ 11 mutant strains, which are capable of responding to odorant cues but fail to be positively imprinted. ${ }^{9}$ Alternatives to our study include possible future research, such as methods involving successful imprinting in $C$. elegans after four generations ${ }^{16}$ as an epigenetic approach to memory, which would allow a new course to continue with our work in the future.

We demonstrated that cryoprotectants used in both the slow freezing and vitrification processes do not affect, alter, or change the mechanism that regulates the olfactory imprinting and long-term memory (Fig. 1). Second, we determined that the process of cryopreservation methods of slow freezing or vitrification do not affect, alter, or change this mechanism (Fig. 1). We also demonstrated that the results of the MI average obtained for the trained and untrained worms are similar to the results of the original authors of the odorant imprinting protocol. ${ }^{9}$

It is essential to emphasize the storage time in liquid nitrogen and the storage time in $\mathrm{a}-80^{\circ} \mathrm{C}$ freezer. In the vitrification protocol, we maintained the worms for $30 \mathrm{~min}$ in liquid nitrogen before warming and for 2 weeks for slow freezing. For worms stored at $-80^{\circ} \mathrm{C}$ in the cryopreservation by slow freezing at the L1 stage, the time between olfactory imprinting and odorant chemotaxis assays was 5 days, although the worms were stored at $-80^{\circ} \mathrm{C}$ for 2 weeks. The worms have a life span of 15 days and develop from first larval stage (L1) to the mature adult stage in approximately $48 \mathrm{hr}^{5}$

There are several differences between the effects of vitrification and those of slow freezing in C. elegans. The most important are the survival rate and the quality of the biological material cryopreserved. The method of vitrification by SafeSpeed for $C$. elegans offers a clear alternative to the traditional protocol by slow freezing due to the high survival rate $(100 \%$ versus $25 \%-35 \%)$ and permits the cryopreservation of all stages of larvae, including the adult stage. ${ }^{31}$ However, from the standpoint of preservation of the memory, the two protocols are equal. Although the value of the MI for trained worms that were cryopreserved by slow freezing (S10) was slightly lower than the MI obtained for trained and vitrified worms (S6) (Table 1), there was no significant difference between the studies.

Whether this result reflects the resilience of structural synaptic change or a more fragile chemical state change to the insult of cryopreservation remains unclear. More detailed chemotaxis assays of learning and memory in cryopreserved $C$. elegans are needed to determine whether all memory mechanisms are unaffected. Further research using methods for learning at different stages of $C$. elegans development to test memory retention after cryopreservation is needed. Additionally, further research on larger organisms with more complex nervous systems, such as the fruit fly Drosophila melanogaster, could prove to be beneficial to the issue of cryopreservation, including, specifically, memory retention after reviving.

\section{Conclusion}

This study introduced the $C$. elegans nematode to olfactory imprinting for learning with the specific goal of testing the animal's memory retention after cryopreservation and revival. Our results show that the mechanisms that regulate odorant imprinting (a form of long-term memory) in young $C$. elegans have not been modified by either the process of vitrification or by slow freezing in the adult stage. This is the first evidence of preservation of memory after cryopreservation (vitrification or slow freezing).

\section{Acknowledgments}

We thank Alcor Research Center (ARC) and Alcor Life Extension Foundation for the grant to develop this study, and Dr. Gregory Fahy and $21^{\text {st }}$ Century Medicine for guidance. We also thank Dr. Max More, President and CEO of Alcor Life Extension Foundation, and Alcor colleagues Hugh Hixon, Steve Graber, and Dr. R. Michael Perry for their insights and support. We thank Dr. Ramon Risco at the University of Seville, and the Caenorhabditis Genetics Center (University of Minnesota, Minneapolis) for wildtype (N2 var Bristol) C. elegans and the University of Advancing Technology for video editing facilities of our research's documentation.

\section{Author Disclosure Statement}

No conflicting financial interests exist.

\section{References}

1. Brenner S. Nobel lecture. Nature's gift to science. Biosci Rep 2003;23:225-237.

2. Brenner S. The genetics of Caenorhabditis elegans. Genetics 1974;77:71-94. 
3. Nass R, Miller III D, Blakely R. C. elegans: A novel pharmacogenetic model to study Parkinson's disease. Parkinsonism Relat Disord 2001;7:185-191.

4. Wu Y, Luo Y. Transgenic C. elegans as a model in Alzheimer's research. Curr Alzheimer Res 2005;2:37-45.

5. Riddle DL, Blumenthal T, Meyer B, Priess J. C. elegans II, 2nd ed. Cold Spring Harbor Laboratory Press, Cold Spring Harbor, New York, 1997.

6. Rankin CH, Beck C, Chiba CM. Caenorhabditis elegans: A new model system for the study of learning and memory. Behav Brain Res 1990;37:89-92.

7. Mah KB, Rankin $\mathrm{CH}$. An analysis of behavioral plasticity in male Caenorhabditis elegans. Behav Neural Biol 1992; 58:211-221.

8. Dunn NA, Lockery SR, Pierce-Shinomura JT, Connery JT. A neural network model of chemotaxis predicts function of synaptic connections in the nematode Caenorhabditis elegans. J Computational Neurosci 2004;17:137-147.

9. Remy JJ, Hobert O. An interneuronal chemoreceptor required for olfactory imprinting in C. elegans. Science 2005; 309:787-790.

10. Ardiel EL, Rankin CH. An elegant mind: Learning and memory in Caenorhabditis elegans. Learn Mem 2010;17: 191-201.

11. Amano H, Maruyama IN. Aversive olfactory learning and associative long-term memory in Caenorhabditis elegans. Learn Mem 2011;18:654-665.

12. Kauffman A, Parsons L, Stein G, Wills A, Kaletsky R, Murphy C. C. elegans positive butanone learning, shortterm, and long-term associative memory assays. J Vis Exp 2011;pii:2490.

13. Sasakura H, Mori I. Behavioral plasticity, learning, and memory in C. elegans. Curr Opin Neurobiol 2013;23:92-99.

14. Gallardo M, Hebles M, Migueles B, Dorado M, Aguilera L, González M, Piqueras P, Montero L, Sánchez-Martín P, Sánchez-Martín F, Risco R. Thermal and clinical performance of a closed device designed for human oocyte vitrification based on the optimization of the warming rate. Cryobiology 2016;73:40-46.

15. Horn G. Memory, Imprinting, and the Brain: An Inquiry into Mechanisms. Claredon Press, Oxford, 1985.

16. Remy JJ, Stable inheritance of an acquired behavior in Caenorhabditis elegans. Curr Biol. 2010;20:877-878.

17. Mazur P. Freezing of living cells: Mechanisms and implications. Am J Physiol 1984;247(3 Pt 1):C125-C142.

18. Fahy GM, MacFarlane DR, Angell CA, Meryman HT. Vitrification as an approach to cryopreservation. Cryobiology 1984;21:407-426.

19. Risco R, Elmoazzen H, Doughty M, He X, Toner M. Thermal performance of quartz capillaries for vitrification. Cryobiology 2007;55:222-229.

20. Fahy GM, Wowk B, Pagotan R, Chang A, Phan J, Thomson $\mathrm{B}$, Phan L. Physical and biological aspects of renal vitrification. Organogenesis 2009;5:167-175.

21. Vajta G, Kuwayama M. Improving cryopreservation systems. Theriogenology 2006;65:236-244.

22. Cobo A, Bellver J, Domingo J, Pérez S, Crespo J, Pellicer A, Remohí J. New options in assisted reproduction tech- nology: The Cryotop method of oocyte vitrification. Reprod Biomed Online 2008;17:68-72.

23. Hamawaki A, Kuwayama M, Hamano S. Minimum volume cooling method for bovine blastocyst vitrification. Theriogenology 1999;51:165.

24. Vajta G, Holm P, Kuwayama M, Booth PJ, Jacobsen H, Greve T, Callesen H. Open pulled straw (OPS) vitrification: A new way to reduce cryoinjuries of bovine ova and embryos. Mol Reprod Dev 1998;51:53-58.

25. He X, Park, EYH, Flower, A, Yarmush ML, Toner, M. Vitrification by ultra-fast cooling at low concentration of cryoprotectants in a quartz microcapillary: A study using murine embryonic stem cells. Cryobiology 2008;56:223232.

26. Risco R, Toner M. Quartz microcapillaries for C. elegans vitrification. Cryobiology 2008;57:320-321.

27. Seki S, Mazur P. Effect of warming rate on the survival of vitrified mouse oocytes and on the recrystallization of intracellular ice. Biol Reprod 2008;79:727-737.

28. Seki S, Mazur P. The dominance of warming rate over cooling rate in the survival of mouse oocytes subjected to a vitrification procedure. Cryobiology 2009;59:75-82

29. Seki S, Mazur P. Ultra-rapid warming yields high survival of mouse oocytes cooled to -196 degrees $C$ in dilutions of a standard vitrification solution. PLoS One 2012; 7:e36058.

30. Mazur P, Seki S. Survival of mouse oocytes after being cooled in a vitrification solution to -196 degrees $\mathrm{C}$ at 95 degrees to 70,000 degrees $\mathrm{C} / \mathrm{min}$ and warmed at $610 \mathrm{de}$ grees to 118,000 degrees $\mathrm{C} / \mathrm{min}$ : A new paradigm for cryopreservation by vitrification. Cryobiology 2011; 62:1-7.

31. Risco R, Hebles M, Saa A, Vilches-Ferron MA, SanchezMartin P, Lucena E, Lucena M. Safespeed technology-the myth of ultrahigh cooling rates: A close device and a serum-free media for the vitrification human oocytes/ embryos with the highest recovery rates. European Society of Human Reproduction and Embryology 29th Annual Meeting 2013;28:180-180.

32. Stiernagle T. Maintenance of $C$. elegans. In: Hope IA, ed. C. elegans: A practical approach. Oxford University Press, Oxford, 1999, pp. 51-67.

33. Gallardo M, Saenz J, Risco R. Human oocytes and zygotes are ready for ultra-fast vitrification after 2 minutes of exposure to standard CPA solutions. Sci Rep. 2019; 9:15986. doi: 10.1038/s41598-019-52014-x. PMID: 31690725; PMCID: PMC6831692.

Address correspondence to: Natasha Vita-More

6729 East Voltaire Avenue Scottsdale, AZ 85254

E-mail: natasha@natasha.cc

Received: October 31, 2014 Accepted: April 11, 2015 